\title{
Collision-Free Operation in Wireless Ad-Hoc Networks
}

\author{
Jaume Barcelo $^{1}$, Boris Bellalta ${ }^{2}$, Miquel Oliver $^{2}$, and Albert Banchs ${ }^{1}$ \\ 1 Universidad Carlos III de Madrid, \\ Av. de la Universidad, 30, 28911 Leganés, Madrid, Spain \\ \{jbarcelo, banchs\} @it.uc3m.es \\ 2 Universitat Pompeu Fabra, \\ C. de Tànger 122-140, 08018, Barcelona, Catalunya, Spain \\ \{boris.bellalta, miquel.oliver\} @upf.edu
}

\begin{abstract}
In some wireless ad-hoc networks it is not possible to rely on carrier-sense mechanisms to prevent collisions. In this article we suggest a MAC protocol that reaches collision-free operation in sparse ad-hoc wireless networks when all the stations are saturated. The basic idea is to use a random backoff after failed transmissions and a deterministic backoff after successful transmissions. Each of the participating stations can configure its own backoff parameter after collecting information from its neighborhood. Then the system enters in a transient-state until collisionfree operation is reached. We assess the duration of the transient-state and other performance metrics for an example scenario and finally we discuss two options to incorporate reception acknowledgements.
\end{abstract}

Keywords: medium access control, wireless ad-hoc networks, collisionfree operation

\section{Introduction and motivation}

Wireless local area networks (WLANs) can be found in homes, campuses, public buildings and enterprises. They are a convenient broadband last-hop alternative, specially for portable and mobile devices. Most of current deployments consist of one or several wireless access points that are connected to a wired network.

Nevertheless, there is substantial interest in extending the coverage of such wireless networks by means of multi-hop wireless links. As an example, multihop can be used to provide coverage on the streets. The manufacturers offer rough outdoor access points that can be placed at light poles. Light poles have power supply but no network connection, and therefore the access points have to create a mesh network to provide connection to the Internet.

Another example of the use of multi-hop wireless networks are grassroots community networks. Some of these networks support thousands of users and consist of thousands of mesh nodes that span over a geographical area of several hundreds of kilometers. This alternative is particularly attractive in rural areas where ADSL is expensive or non-existent [1]. 
Most of the currently deployed mesh networks, which belong to the broad family of ad-hoc networks, use WLAN hardware due to its availability and affordability. As a result, WLAN protocols are also used in mesh networks. The promise of highly-configurable WLAN firmware [2] and the possibility to develop tailored medium access control (MAC) protocols for different kinds of networks motivates us to explore a simple MAC protocol that is appropriate for ad-hoc networks.

In this article we study some of the characteristics of ad-hoc networks that make them intrinsically different from wireless WLANs. In particular, in Sec. 2 we describe three specific problems that arise in ad-hoc networks. These challenges warrant the study of new protocols which are tailored to the distinctive properties of ad-hoc networks. In Sec. 2, we also briefly mention those works that are closely related and relevant for the subsequent discussion, and we define the scope and the goal of our work. We are interested in a distributed protocol that can achieve collision-free operation in ad-hoc networks, without requiring network-wide synchronization.

The protocol itself is described in Sec. 3 and relies on a very simple idea: the use of a deterministic backoff after successful transmissions and a random backoff after failures. In commonly encountered topologies, this approach guarantees that collision-free operation can be reached in a finite time and the network converges to a periodic, deterministic collision-free schedule.

Then, we propose a distributed approach that allows each node to configure its own contention parameter in Sec. 4. In Sec. 5, we use a simple example scenario to explain that there is a trade-off that involves the duration of the transient-state and the efficiency that it is obtained in the steady-state. Sec. 6 is devoted to a protocol comparison that includes different performance metrics. Two different options to accommodate the acknowledgements of correct receptions are outlined in 7 and finally some conclusions remarks are offered in 8.

\section{State-of-the-Art and Open Challenges}

One of the limitations of several wireless communications systems is that it is not possible for the terminals to transmit and receive simultaneously. A receiver may also have difficulties decoding several transmissions from different senders that are transmitted simultaneously. When a message arrives to a receiver while there is already an ongoing transmission or reception, we say that a collision has occurred. A collision results in the loss of one or more the incoming colliding messages.

In the design and study of MAC protocols for WLANs, it is common to assume that all the different stations are in each other's transmission range. This is a fair assumption since WLANs are often confined to a reduced area. Under this assumption, the contending stations can rely on carrier-sense mechanisms to avoid collisions. If the participating stations transmit only when the channel is sensed idle, the likelihood of collisions is greatly reduced. In general, the avail- 
ability of carrier-sense information allows for high channel efficiency in the case of carrier sense multiple access with collision avoidance (CSMA/CA).

Unfortunately, the assumption that all the stations can hear all the transmissions is no longer valid in multi-hop wireless networks. The reason that we need multi-hop is, very often, the fact that there are at least two stations that do not hear each other and therefore they need the help of the other stations to relay the messages in a multi-hop fashion. Even for those stations that can hear each other, carrier sense may fail due to long propagation times. This is the case of long radio-links typically used in rural community wireless networks, that can easily be in the order of tens of kilometers with the help of directive antennae.

Unsurprisingly, the absence of carrier-sense information breaks the operation of CSMA/CA. We will provide three examples of the undesirable effects of using a CSMA/CA protocol as the one included in the IEEE 802.11 [3] suite of standards.

1. Hidden terminal effect: This problem arises when an interferer cannot carriersense the transmitter as illustrated in Fig. 1.(a). More details on the hidden terminal effect and its negative interaction with the upper layers of the protocol stack in a real mesh network deployment can be found in [4].

2. Exposed terminal effect: as illustrated in 1.(b), two senders $B$ and $C$ want to transmit to two receivers $A$ and $D$ respectively. $B$ and $C$ are in each other's transmission range, but their transmissions do not interfere because $A$ is much closer to $B$ than it is to $C$ and, similarly, $B$ and $D$ are far away. In this situation the simultaneous transmission of $B$ and $C$ would not result in a collision. Still, the carrier-sense mechanism prevents the simultaneous transmission of $B$ and $C$.

3. Fig. 1.(c) shows two stations, $E$ and $F$ that are far away. Still, they can communicate thanks to the use of directional antennae. Even though they are in each other's transmission range, the propagation delay interferes with the carrier-sense mechanism. The propagation delay introduces a vulnerability interval in which one of the stations is transmitting while the other senses the channel idle. More details on the impact of large propagation delay on the performance of IEEE 802.11 can be found in [5].

As a consequence of the above described effects, it is apparent that a carriersense medium access control is not appropriate when carrier-sense information is not available or reliable. For this reason, many mesh network deployments have resorted to multi-channel solutions. When budget and spectrum availability is not a concern, the first two of the aforementioned problems (hidden and exposed terminal) can be trivially solved by assigning a different channel to each radiolink. The third problem may still increase the probability of collision, but it is a minor issue if the distance is not too long.

Assigning different channels to different links requires that each station is equipped with multiple radios, which increase the price of the device. In this paper we will study the (challenging) case in which, due to either budgetary or spectrum constraints, each of the stations is equipped with a single radio, and the three problems of multi-hop wireless communications (hidden, exposed and distant terminal) need to be addressed at the MAC layer. 


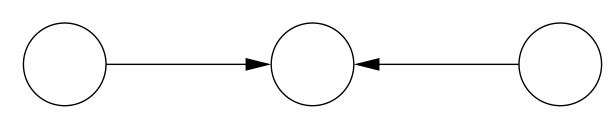

(a)
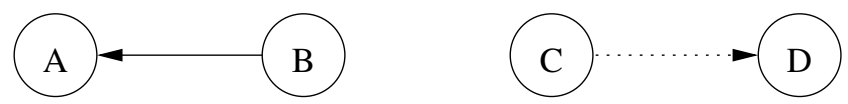

(b)

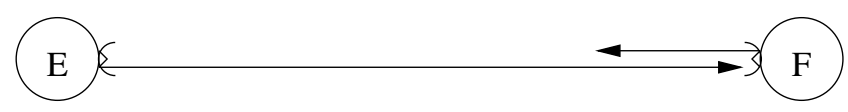

(c)

Fig. 1. Problems arising when using CSMA in ad-hoc and long-distance wireless networks. (a) the hidden terminal effect. (b) the exposed terminal effect. (c) the distant terminal effect.

A survey on modeling and performance analysis of multihop packet radio networks is presented in [6]. A theoretical advance was presented in [7] where it was shown that it was possible to analyze the problem of offering proportional fairness in ad-hoc aloha networks using local information. Under certain assumptions, the transmission rate of each station can be computed by knowing some information about the neighbors and the two-hop neighbors. This approach is valid for both slotted aloha and pure aloha networks. In slotted aloha, it is required that all the stations synchronize to the beginning of the slots.

The possibility of collision-free operation has been studied in, e.g., [8-11]. All these references assume that the network time is slotted, and some of them refer to those protocols that can potentially learn a collision-free schedule as learning protocols. In the present work we present two protocols that can learn a collisionfree schedule even in the absence of slot synchronization. The first one, l-aloha, assumes previous knowledge of the topology and communication graph. In the second one, scl-aloha, the nodes can self-configure using information collected from their neighborhood.

\section{L-Aloha: Deterministic Backoff After Successes in Ad-Hoc Networks}

In this section we will propose a protocol that can achieve collision-free operation in ad-hoc networks where carrier-sense information is incomplete or unavailable. For the sake of tractability, we will rely on several simplifications:

- We will ignore the complexities of radio propagation and assume a graphbased interference model. I.e., two nodes perfectly hear each other or they do not hear each other at all (ideal channel). 
- There is no capture effect. It is not possible to decode overlapping packets.

- The stations are saturated, which means that they always have a packet ready to be transmitted.

- The duration of a transmission is fixed and normalized to one. The protocol that we are suggesting in this paper can also be extended to work in networks where the packet length is variable and upper-bounded.

- Initially, acknowledgement packets will not be explicitly considered. This discussion is postponed to Sec. 7 .

Since we use a graph-based interference model, we can represent the topology of the network as an undirected graph $G=(S, L)$ where $S$ represents the set of stations and $L$ is the set of links. If a link exists between two stations $s_{i}$ and $s_{j}$ in $S$, then these two stations can communicate with each other and also interfere with each other. We define the set of neighbors $K_{i}$ of a station $s_{i}$ as those stations that have a link connecting to $s_{i}$, i.e. $K_{i}=\left\{s_{j}:\left(s_{i}, s_{j}\right) \in L\right\}$.

The fact that two stations are in each other's transmission range does not necessarily mean that they are exchanging data. We will say that there is a data flow between a station $s_{i}$ and a station $s_{j}$ when $s_{i}$ is transmitting data to $s_{j}$. This is a directed edge $\left(s_{i}, s_{j}\right)$ between the two stations and we call the set of all flows $F$.

We will use the simple topology presented in Fig. 2 for exemplifying purposes. In this topology we have three different stations $\left(S=\left\{s_{1}, s_{2}, s_{3}\right\}\right)$, two links $\left(L=\left\{l_{1}, l_{2}\right\} ; l_{1}=\left(s_{1}, s_{2}\right) ; l_{2}=\left(s_{2}, s_{3}\right)\right)$ and three flows $\left(F=\left\{f_{1}, f_{2}, f_{3}\right\} ; f_{1}=\right.$ $\left.\left(s_{1}, s_{2}\right) ; f_{2}=\left(s_{2}, s_{1}\right) ; f_{3}=\left(s_{3}, s_{2}\right)\right)$.

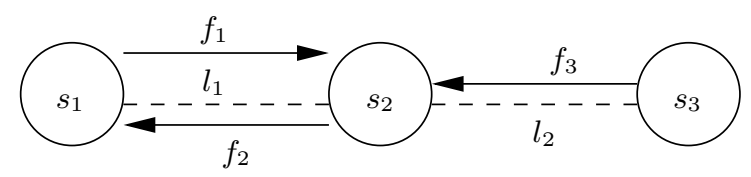

Fig. 2. Topology of a simple ad-hoc network.

From the topology description in Fig. 2 we can derive the interference graph in Fig. 3. This is a directed graph where and edge from $f_{2}$ to $f_{3}$ means that a transmission of $f_{3}$ will be lost if it overlaps in time with a transmission from $f_{2}$. Contrastingly, the transmission in $f_{2}$ is unaffected by the transmission of $f_{3}$.

In this example, the interference graph is strongly connected. This means that the graph contains a directed path from $f_{i}$ to $f_{j}$ and a directed path from $f_{j}$ to $f_{i}$ for any pair of vertices $f_{i}$ and $f_{j}$.

For given set of flows, a schedule $\sigma$ defines which of the flows are active at any given time. The period of the schedule is $T_{\sigma}$ and we are interested in a collision-free schedule that satisfies the following conditions:

- When a node is active, the destination node must be silent, i.e. $s_{k}$ must be silent when flow $f_{i}=\left(s_{j}, s_{k}\right)$ is active. 


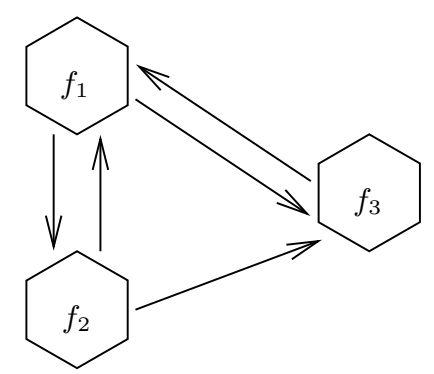

Fig. 3. Interference graph of a simple ad-hoc network.

- The neighbors of the destination node must also be silent for the duration of the transmission. I.e, when $f_{i}=\left(s_{j}, s_{k}\right)$ is active, all nodes belonging to $K_{k}$ must remain silent.

For any given topology, it exists a minimum schedule period which we call $T_{\sigma}^{\text {min }}$ that can accommodate a collision-free schedule. In our particular example in Fig. 3, $T_{\sigma}^{\text {min }}=3$ because there are three flows and the simultaneous transmission of any two flows would result in collision. We suggest a distributed protocol that satisfies that, for any topology with a strongly connected interference graph, and a given schedule duration $T_{\sigma}$ which is strictly greater than $T_{\sigma}^{\text {min }}$, a collisionfree schedule is reached in a finite time. We will use a value $T_{\sigma}=T_{\sigma}^{\min }(1+\epsilon)$ where $\epsilon$ is a positive real value arbitrarily close to one.

The idea is to use a random backoff after failed transmissions and a deterministic backoff after successful transmissions. The random backoff is exponentially distributed with parameter $\lambda=\left(T_{\sigma}\right)^{-1}$. The random backoff starts to count at the end of the unsuccessful transmission. The deterministic backoff after successes is equal to $T_{\sigma}$, with the particularity that in this case the backoff time starts to count at the beginning of the successful transmission. In other words, $T_{\sigma}$ is the time elapsing from the beginning of a successful transmission to the beginning of the next transmission attempt. The goal is that, in the collisionfree mode of the operation, the behaviour of each station is periodic with period equal to $T_{\sigma}$.

This is a key difference from our previous work in slotted networks and therefore deserves some additional explanation. Since the network that we are considering in the present paper is not slotted, the backoff period (and the length of the schedule) is continuous and has to be expressed in units of time, not in slots. Moreover, the time elapsing between a successful transmission and the next transmission has to be independent of the packet length. This is an important property that makes it possible to extend our approach to networks in which the packet length is variable.

We will use Fig. 4 for exemplifying purposes. It represents two stations that run our proposed protocol and the rounded shapes are the stations' transmissions. The two stations collide in their first transmission attempt and therefore compute an exponentially distributed backoff that is measured from the end of 
a transmission to the beginning of the next transmission. The two stations successfully transmit in their next transmission attempt, and consequently choose a deterministic backoff value that starts at the beginning of the successful transmission. At this point the behaviour of the system is completely deterministic. Note that in the hypothetical case that one of the stations transmitted a shorter packet after reaching collision-free operation, the duration of the packet transmission would not affect the global schedule.

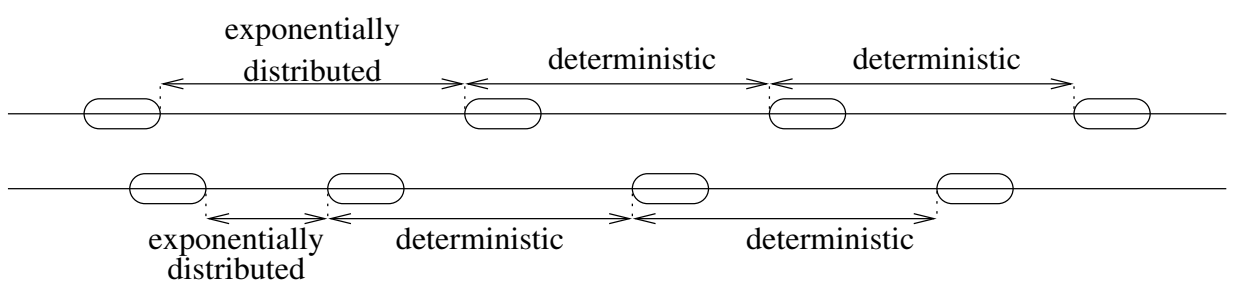

Fig. 4. A deterministic backoff is used after successful transmissions and a random backoff is used after collisions.

In a topology with a strictly connected interference graph, any station that is not settled in a collision-free schedule can trigger a chain of collisions that can potentially reach all the other stations. All the stations that suffer a collision will choose their backoff at random and there is a finite possibility that they choose a global collision-free schedule.

\section{Scl-Aloha: Distributed Self-Configuration}

An attractive property of the solution presented in [7] is the possibility of distributed self-configuration. In that reference, the transmission rate of each aloha station can be computed using locally gathered information. The idea is that each station broadcasts some hello messages containing information about the number of incoming and outgoing flows. Each node collects the hello messages from its neighbors and uses this information to decide its own transmission rate. The advantage is that a node does not need to know the whole network topology in order to self-configure.

In this section we suggest a similar self-configuration approach that will make it possible to reach collision-free operation using the information provided by neighboring nodes. We use the name scl-aloha to refer to this self-configuring learning aloha. Each node $i$ gathers information about its number of incoming flows $\left|I_{i}\right|$ and outgoing flows $\left|O_{i}\right|$ and distributes this information to its immediate neighbors using broadcast hello messages. Therefore, each station knows the number of incoming flows of each of its neighbors and also its number of incoming flows. At, this point, each station $s_{i}$ computes the duration of its schedule as:

$$
T_{\sigma_{i}}=2^{\left\lceil\log _{2}\left(\sum_{k \in K_{i}}\left|I_{k}\right|+\left|O_{k}\right|\right)\right\rceil}(1+\epsilon),
$$


which is the smallest power of two that it is larger than the sum of all flows in the one-hop neighborhood multiplied by a number which is slightly larger than one. The $\lceil\cdot\rceil$ operator is the ceiling operator. And the value of $\epsilon$ has to be common for all the stations of the network.

With this approach, it is possible that different nodes obtain a different schedule length. The global schedule $T_{\sigma}$ is the largest of all $T_{\sigma_{i}}$. Those stations that use a schedule which is an integer fraction of the global schedule, will transmit multiple times in each global schedule.

The performance of scl-aloha is compared to l-ahola in Table 1 in Sec. 6. Since the global schedule is restricted to be a power of two multiple of $(1+\epsilon)$, the length of the schedule that we obtain with scl-aloha is longer than in l-aloha (for a fixed value of $\epsilon$ ). Therefore, the performance metrics that are achieved by scl-aloha in the steady-state are not as good as the ones that can be attained with l-aloha. Nevertheless, the use of a longer schedule has the advantage of having a much shorter transient-state duration, as we will see in the next section.

\section{Transient State Duration}

The learning protocols presented in the present article require some time to reach collision-free operation, which is the steady-state. The time to reach collision-free operation is a random variable and its distribution depends on the topology. We will analyze the same topology that we have already discussed in Sec. 3 and we will use simulations to measure the average time that is required for the system to reach collision-free operation.

We have used a custom simulator in c (the source code is available upon request) that implements only the MAC layer. The simulator adopts all the assumptions that we have used in our previous discussion of the protocol. Averages are computed across 100,000 simulation runs and the standard error of the mean is below $5 \%$.

The schedule length $T_{\sigma}$ is the time that elapses from the start of a successful transmission to the start of the following transmission. This very same value is the parameter of the exponential random distribution that is used to choose the random backoff after a failed transmission. Remember that the random backoff time starts at the end of an unsuccessful transmission, while the deterministic backoff is measured from the beginning of the successful transmission (See Fig. 4).

In our example scenario, the schedule length $T_{\sigma}$ has to be larger than three in order to accommodate three stations transmitting in a collision-free fashion. Therefore, we take schedule length values from 3.25 up to 15.75 and we evaluate two performance metrics: the steady-state aggregated traffic and the time required to reach collision-free operation.

The results are presented in Fig. 5, which shows that our two metrics of interest decrease as we increase the schedule length $T_{\sigma}$. Therefore, there is a design trade-off which is controlled by the choice of the schedule length or, equivalently, the parameter $\epsilon$ in Table 1 in the next section. If we want to attain 
a high aggregated throughput in the steady-state, we should be ready to accept a long transient-state.

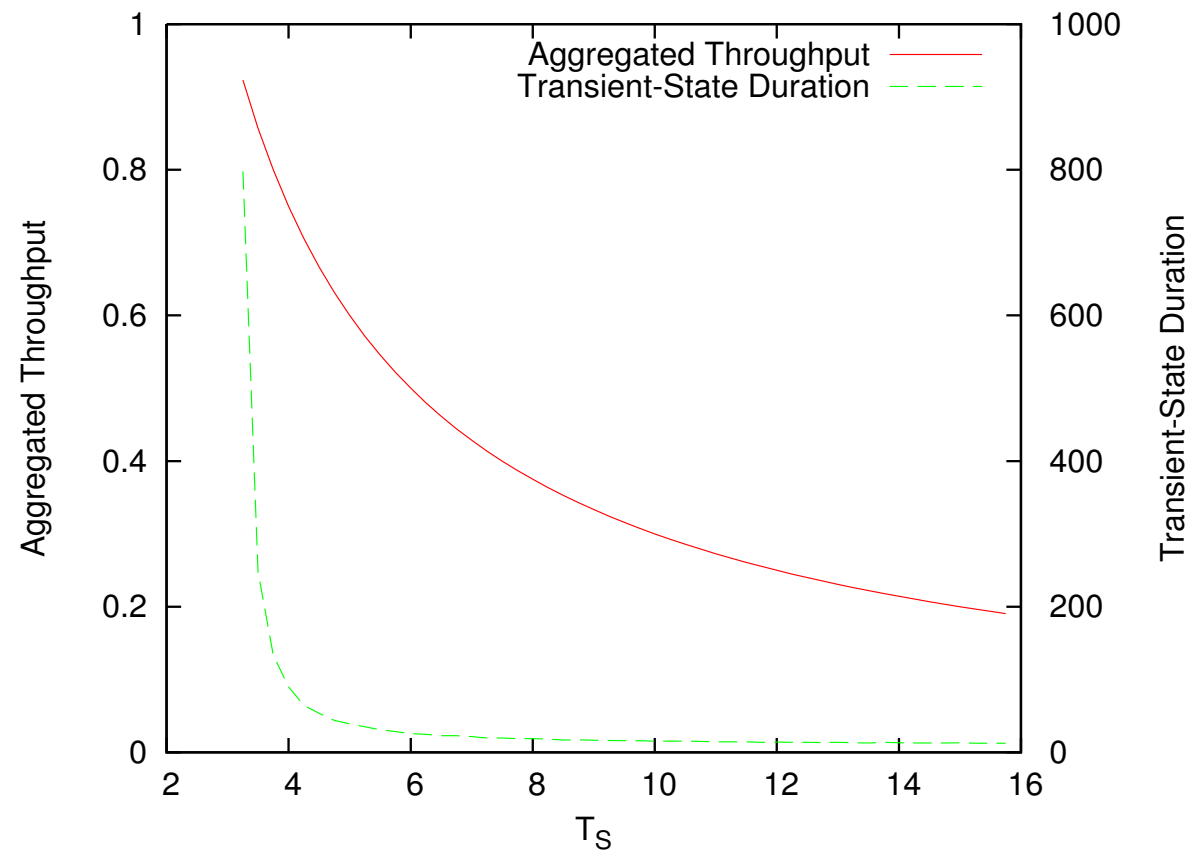

Fig. 5. This plot shows the aggregated throughput and the time required to reach collision-free operation in the example topology of Fig. 2 for different values of the schedule length $T_{\sigma}$.

\section{A Performance Comparison}

This section compares the steady-state performance of our proposed protocols (l-aloha, scl-aloha) with the performance of aloha. The results are summarized in Table 1, where log denotes the natural logarithm.

The first three columns $\left(\tau_{1}, \tau_{2}, \tau_{3}\right)$ show the transmission rate of the three stations, which is the average fraction of time that each of the stations devotes to transmission. The transmission rate of the aloha protocol has been computed in such a way that maximizes proportional fairness [7]. The transmission rate for l-aloha and scl-aloha is simply $\frac{1}{T_{\sigma}}$. Note that knowledge of the global topology is assumed in aloha and $T_{\sigma}=3(1+\epsilon)$. In scl-aloha, the schedule length is locally computed and we obtain $T_{\sigma}=4(1+\epsilon)$.

The following three columns $\left(\theta_{1}, \theta_{2}, \theta_{3}\right)$ represent the throughput attained by each of the stations, which is the time devoted to successful transmission by each 
of the stations. The derivation of the throughput for optimum aloha is detailed in the Appendix.

The last three columns represent three different performance metrics: Jain's Fairness index $\left(J F=\frac{\left(\theta_{1}+\theta_{2}+\theta_{3}\right)^{2}}{3\left(\theta_{1}^{2}+\theta_{2}^{2}+\theta_{3}^{2}\right)}\right)$, aggregated throughput $\left(A T=\theta_{1}+\theta_{2}+\theta_{3}\right)$ and proportional fairness $\left(P F=\log \theta_{1}+\log \theta_{2}+\log \theta_{3}\right)$.

It is remarkable that, for a sufficiently small value of $\epsilon$, l-aloha outperforms aloha in the three metrics that are considered in this particular comparative. We can observe that l-aloha achieves perfect fairness and that, by taking a sufficiently small value of $\epsilon$, the aggregate throughput can be increased to a value arbitrarily close to one.

Table 1. Performance Comparison

\begin{tabular}{|c||c|c|c|c|c|c|c|c|c|}
\hline \multirow{2}{*}{ Contention } & \multicolumn{3}{|c|}{ Tx rate } & \multicolumn{3}{c|}{ Throughput } & \multicolumn{3}{c|}{ Performance } \\
\hline & $\tau_{1}$ & $\tau_{2}$ & $\tau_{3}$ & $\theta_{1}$ & $\theta_{2}$ & $\theta_{3}$ & JF & AT & PF \\
\hline \hline aloha & $\frac{\sqrt{\frac{3}{2}}-1}{\sqrt{\frac{3}{2}}}$ & $\frac{\sqrt{\frac{3}{2}}-1}{\sqrt{\frac{3}{2}}}$ & $\frac{\sqrt{2}-1}{\sqrt{2}}$ & 0.056 & 0.120 & 0.108 & 0.921 & 0.283 & -7.234 \\
l-aloha & $\frac{1}{3(1+\epsilon)}$ & $\frac{1}{3(1+\epsilon)}$ & $\frac{1}{3(1+\epsilon)}$ & $\frac{1}{3(1+\epsilon)}$ & $\frac{1}{3(1+\epsilon)}$ & $\frac{1}{3(1+\epsilon)}$ & 1 & $\frac{1}{1+\epsilon}$ & $-3.296-3 \log (1+\epsilon)$ \\
scl-aloha & $\frac{1}{4(1+\epsilon)}$ & $\frac{1}{4(1+\epsilon)}$ & $\frac{1}{4(1+\epsilon)}$ & $\frac{1}{4(1+\epsilon)}$ & $\frac{1}{4(1+\epsilon)}$ & $\frac{1}{4(1+\epsilon)}$ & 1 & $\frac{3}{4(1+\epsilon)}$ & $-4.159-3 \log (1+\epsilon)$ \\
\hline
\end{tabular}

\section{Reception Acknowledgements}

In order to make it possible to compare our protocol with Aloha, we have not discussed the presence of acknowledgements in the previous sections. Nevertheless, acknowledgements play a critical role in wireless networks in general, and in our proposed protocols in particular. Both l-aloha and scl-aloha use a deterministic backoff after successful transmissions and a random backoff otherwise. Therefore the stations need to know whether a transmission has been successful or not. It should be noted that the acknowledgement is transmitted in the reverse direction of the data transmission, and therefore the interference graph for the acknowledgements is different than the one for data packets.

There are two possible approaches to accommodate acknowledgements, and both of them have some detrimental effect on performance. The first approach is to modify Eq. 1 to account for the flows of two-hop neighbors to guarantee that a collision-free schedule also exists in the reverse path. As a result, the schedule will be longer and the steady-state performance will be lower. A second approach is to require all the flows to be bidirectional and piggyback the acknowledgements in data packets. This solution is much more efficient in the steady-state but 
requires a longer transient state since the random backoff has to be longer than $T_{\sigma}$ to give time to the correspondent node to transmit its data packet.

\section{Conclusions}

In this paper we have studied the possibility of reaching collision-free operation in ad-hoc networks by using a deterministic backoff after successful transmissions. We have considered a simple example scenario in which collision-free operation results in a performance improvement when the collision-free schedule is learned by all nodes after a transient-state. Finally, we have proposed a mechanism that the stations can use to self-configure their contention parameter using information that is gathered from the neighboring nodes.

\section{Acknowledgements}

V. Mancuso provided several corrections and ideas to improve an earlier version of the manuscript. This work has been partially supported by the Spanish Government (TEC2008-0655, Plan Nacional I+D), (CSD2008-00010, ConsoliderIngenio Program) and by the Catalan Government (SGR2009\#00617).

\section{References}

1. Oliver, M., Zuidweg, J., Batikas, M.: Wireless Commons Against the Digital Divide. In: IEEE International Symposium on Technology and Society ISTAS, New South Wales, Australia (2010)

2. Gringoli, F., Nava, L.: OpenFWWF: OpenFirmWare for WiFi networks. http://www.ing.unibs.it/ openfwwf/ (2010) [Online; accessed 25-March-2011].

3. IEEE 802.11: Wireless LAN Medium Access Control (MAC) and Physical Layer (PHY) Specification (2007)

4. Gurewitz, O., Mancuso, V., Shi, J., Knightly, E.: Measurement and Modeling of the Origins of Starvation of Congestion-Controlled Flows in Wireless Mesh Networks. IEEE/ACM Transactions on Networking 17(6) (2009) 1832-1845

5. Lopez-Aguilera, E., Casademont, J., Cotrina, J.: Propagation Delay Influence in IEEE 802.11 Outdoor Networks. Wireless Networks 16(4) (2010) 1123-1142

6. Tobagi, F.: Modeling and performance analysis of multihop packet radio networks. Proceedings of the IEEE 75(1) (1987) 135-155

7. Kar, K., Sarkar, S., Tassiulas, L.: Achieving Proportional Fairness Using Local Information in Aloha Networks. IEEE Transactions on Automatic Control 49(10) (2004) 1858-1863

8. He, Y., Yuan, R., Sun, J., Gong, W.: Semi-Random Backoff: Towards Resource Reservation for Channel Access in Wireless LANs. In: IEEE ICNP. (2009) 21-30

9. Barcelo, J., Bellalta, B., Cano, C., Sfairopoulou, A., Oliver, M.: Towards a Collision-Free WLAN: Dynamic Parameter Adjustment in CSMA/E2CA. In: EURASIP Journal on Wireless Communications and Networking. (2011) To appear. 
10. Fang, M., Malone, D., Duffy, K., Leith, D.: Decentralised Learning MACs for Collision-free Access in WLANs. Arxiv preprint arXiv:1009.4386v2 (2011)

11. Yi, Y., De Veciana, G., Shakkottai, S.: MAC Scheduling With Low Overheads by Learning Neighborhood Contention Patterns. IEEE/ACM Transactions on Networking (2010)

\section{Appendix: Aloha Throughput in the Simple Example Ad-Hoc Network}

In this appendix we derive the throughput of the stations 1 to 3 in the example scenario that is presented in Fig. 2. We will use the notation $s_{i}$ for $i \in\{1,2,3\}$ to name the stations. Each station $s_{i}$ transmits fixed-length packets with a transmission duration normalized to one and then backs off for an exponentially distributed time. The parameter of this exponential distribution is $\lambda_{i}$.

We follow the steps in [7] and model the transmissions as a renewal process. The expected time interval that elapses between two consecutive transmission epochs is the sum of the transmission time and the exponentially distributed backoff, totalling $1+1 / \lambda_{i}$. And the throughput obtained by station $s_{i}$ will be computed as $S_{i}=\phi_{i} P_{i}$, where $\phi_{i}$ denotes the fraction of time that station $s_{i}$ devotes to transmissions and $P_{i}$ is the probability that one transmission by station $s_{i}$ succeeds. Since station $s_{i}$ transmits during one unit of time in each transmission epoch, the fraction of time devoted to transmissions is simply $\phi_{i}=\frac{1}{1+\lambda_{i}}$.

Particularizing now for our example scenario, we need that two conditions are satisfied for a transmission by $s_{1}$ to be successful. First, both $s_{2}$ and $s_{3}$ have to be silent when the transmission by $s_{1}$ starts and, second, both $s_{2}$ and $s_{3}$ have to remain silent for one unit of time. The probability that a station $s_{i}$ is silent at any given time is $1-\phi_{i}$ and the probability that a station that is silent remains silent for one unit of time is $e^{-\lambda_{i}}$. Consequently,

$$
P_{1}=\left(1-\phi_{2}\right)\left(1-\phi_{3}\right) e^{-\lambda_{2}} e^{-\lambda_{3}}=\frac{1}{\left(1+\lambda_{2}\right)\left(1+\lambda_{3}\right)} e^{-\left(\lambda_{2}+\lambda_{3}\right)} .
$$

And finally, we can compute the throughput obtained by $s_{1}$ as

$$
S_{1}=\phi_{1} P_{1}=\frac{\lambda_{1}}{1+\lambda_{1}} \frac{1}{\left(1+\lambda_{2}\right)\left(1+\lambda_{3}\right)} e^{-\left(\lambda_{2}+\lambda_{3}\right)} .
$$

We repeat the same steps to compute the throughput of $s_{2}$ (taking into account that only transmissions from $s_{1}$ disrupt transmissions from $s_{2}$ )

$$
S_{2}=\frac{\lambda_{2}}{1+\lambda_{2}} \frac{1}{1+\lambda_{1}} e^{-\lambda_{1}}
$$

and the throughput of $s_{3}$

$$
S_{3}=\frac{\lambda_{3}}{1+\lambda_{3}} \frac{1}{\left(1+\lambda_{2}\right)\left(1+\lambda_{1}\right)} e^{-\left(\lambda_{2}+\lambda_{1}\right)} .
$$

